\title{
Epicardial Adipose Tissue Is Nonlinearly Related to Anthropometric Measures and Subcutaneous Adipose Tissue
}

\author{
Miroslav Šram, ${ }^{1}$ Zvonimir Vrselja, ${ }^{2}$ Igor Lekšan, ${ }^{3}$ Goran Ćurić, \\ Kristina Selthofer-Relatić, ${ }^{1,5}$ and Radivoje Radic ${ }^{2}$ \\ ${ }^{1}$ Department of Cardiology, Clinic of Internal Medicine, Osijek University Hospital Centre, 31000 Osijek, Croatia \\ ${ }^{2}$ Department of Anatomy and Neuroscience, Faculty of Medicine, University of J.J. Strossmayer in Osijek, 31000 Osijek, Croatia \\ ${ }^{3}$ Department of Cardiac Surgery, Clinic of Surgery, Osijek University Hospital, 31000 Osijek, Croatia \\ ${ }^{4}$ DNA Laboratory, Department of Chemistry, Biochemistry and Clinical Chemistry, Faculty of Medicine, University of J.J. Strossmayer \\ in Osijek, 31000 Osijek, Croatia \\ ${ }^{5}$ Department of Internal Medicine, Faculty of Medicine, University of J.J. Strossmayer in Osijek, 31000 Osijek, Croatia
}

Correspondence should be addressed to Kristina Selthofer-Relatić; selthofer.relatic@gmail.com

Received 9 December 2014; Accepted 6 April 2015

Academic Editor: Manuel Estrada

Copyright (C) 2015 Miroslav Šram et al. This is an open access article distributed under the Creative Commons Attribution License, which permits unrestricted use, distribution, and reproduction in any medium, provided the original work is properly cited.

Introduction. Adipose tissue is the largest endocrine organ, composed of subcutaneous (SAT) and visceral adipose tissue (VAT), the latter being highly associated with coronary artery disease (CAD). Expansion of epicardial adipose tissue (EAT) is linked to CAD. One way of assessing the CAD risk is with low-cost anthropometric measures, although they are inaccurate and cannot discriminate between VAT and SAT. The aim of this study is to evaluate (1) the relationship between EAT thickness, SAT thickness and anthropometric measures in a cohort of patients assessed at the cardiology unit and (2) determine predictive power of anthropometric measures and EAT and SAT thickness in establishment of CAD. Methods. Anthropometric measures were obtained from $53 \mathrm{CAD}$ and 42 non-CAD patients. Vascular and structural statuses were obtained with coronarography and echocardiography, as well as measurements of the EAT and SAT thickness. Results. Anthropometric measures showed moderate positive correlation with EAT and SAT thickness. Anthropometric measures and SAT follow nonlinear $S$ curve relationship with EAT. Strong nonlinear power curve relationship was observed between EAT and SAT thinner than 10 mm. Anthropometric measures and EAT and SAT were poor predictors of CAD. Conclusion. Anthropometric measures and SAT have nonlinear relationship with EAT. EAT thickness and anthropometric measures have similar CAD predictive value.

\section{Introduction}

The most common chronic disorder nowadays is obesity, which is defined as excessive accumulation of adipose tissue (fat), traditionally defined with body mass index (BMI) exceeding $30 \mathrm{~kg} / \mathrm{m}^{2}$. The majority of adipose tissues in the human body are deposited as subcutaneous or visceral fat, differing in their structure and function [1]. Subcutaneous adipose tissue (SAT) is primarily located on the extremities, while visceral adipose tissue (VAT) is located around internal organs. Adipose tissue has important endocrine role, as genetic analyses have shown that adipose tissue (especially VAT) expresses numerous secretory proteins (adipocytokines) [2]. Recently it was established that proinflammatory adipocytokines secreted by thickened epicardial adipose tissue (EAT), VAT located around the heart and coronary arteries, lead to the development of CAD [3,4]. Obesity is recognized as risk factor for coronary artery disease (CAD) $[5,6]$. Particularly risk-increasing is abdominal obesity, characterized with predominantly abdominal accumulation of fat (VAT) [7, 8]. Abdominal obesity is key characteristic for establishment of the metabolic syndrome, together with any two of the following: hypertriglyceridemia, reduced high density lipoprotein (HDL), raised blood pressure, and disorders of carbohydrate metabolism (raised fasting plasma glucose or diabetes type 2) [9]. Since BMI is general indicator of obesity, measures of abdominal obesity, such as waist 
circumference (WC), waist-to-hip ratio (WHR), and waistto-height ratio (WHR), are suggested as more accurate in describing the distribution of body fat compared. Ultrasound anthropometric indicators, such as SAT and EAT thickness, have been recently proposed as methods of assessment of body fat distribution. Research on relationships between "classic" and ultrasound anthropometric indicators is limited [10].

Coronary heart disease is the leading cause of death worldwide. Coronary angiography is the criterion standard for detecting significant flow-limiting stenosis and direct imaging of atherosclerotic changes in coronary arteries. Because of the inherent limitations, disadvantages and complications of coronary angiography, attention has been directed toward using physiologic, noninvasive modalities to determine the severity of coronary stenosis. Epicardial adipose tissue thickness has been reported as a marker for the presence and severity of coronary artery disease $[3,4,11]$, superior to WC and WHR.

Due to limited research in the field, we performed the detailed analysis of the relationship between anthropometric measures (WC, hip circumference (HC), WHR, BMI, and waist-to-height ratio) and echocardiographically obtained EAT and SAT thickness in a cohort of patients admitted at the cardiology unit. We also tested power of these "classical" and ultrasound anthropometric measures for the prediction of CAD.

\section{Methods}

2.1. Study Population. In this study 95 Caucasian subjects were included, 55 males and 40 females, all of whom signed an informed consent. Subject's age spanned from 31 to 80 years, with BMI of 19.9 to $38.7 \mathrm{~kg} / \mathrm{m}^{2}$. Due to clinical symptoms all subjects underwent echocardiography and coronarography at the Cardiology Department of Clinical Hospital Center Osijek, Croatia. Exclusion criteria were diabetes and chronic renal failure.

\subsection{Epicardial and Subcutaneous Adipose Tissue Thickness} Measurement. Ultrasound measurements of EAT thickness were performed with Siemens Acuson V70 and linear probe L10 $(5-11 \mathrm{MHz})$ and SAT thickness with probe P4-2 (all Siemens Medical Solutions, Malvern, PA, USA). EAT thickness was assessed using $\mathrm{M}$ mode in long parasternal axis with subject in left lateral decubitus position. The EAT was identified as echo-free space between the myocardial wall and the visceral layer of pericardium in end-diastole above right ventricle $[3,4,11,12]$. Subcutaneous adipose tissue thickness was measured above umbilicus while the subject was on his/her back. All measurements were done in triplicate with probe repositioning by one sonographer.

2.3. Anthropometry. Anthropometric measures were taken in all subjects in standing position, while being barefoot on a flat surface, after exhaling, at a level parallel to the floor (waist
TABLE 1: Anthropometric measures and EAT and SAT thickness in CAD and non-CAD subjects.

\begin{tabular}{lcc}
\hline & CAD & Non-CAD \\
\hline Number & 53 & 42 \\
Age $($ years $)$ & $62 \pm 10$ & $62 \pm 13$ \\
BMI $\left(\mathrm{kg} / \mathrm{m}^{2}\right)$ & $27.90 \pm 3.08$ & $27.03 \pm 5.30$ \\
Waist circumference $(\mathrm{cm})$ & $98.84 \pm 12.19$ & $89.95 \pm 12.82^{*}$ \\
Hip circumference $(\mathrm{cm})$ & $98.96 \pm 9.39$ & $93.32 \pm 12.59^{*}$ \\
Waist-to-hip ratio & $0.99 \pm 0.08$ & $0.96 \pm 0.05^{*}$ \\
Waist-to-height ratio & $0.57 \pm 0.06$ & $0.53 \pm 0.08^{*}$ \\
EAT thickness $(\mathrm{mm})$ & $5.25 \pm 2.50$ & $3.89 \pm 1.76^{*}$ \\
SAT thickness $(\mathrm{mm})$ & $15.38 \pm 6.67$ & $12.32 \pm 8.33^{*}$ \\
\hline
\end{tabular}

CAD: coronary artery disease; BMI: body mass index; EAT: epicardial adipose tissue; SAT: subcutaneous adipose tissue. ${ }^{*} p<0.05$ : difference between the groups.

circumference, hip circumference, height), using a stretchresistant measuring tape to the nearest $1 \mathrm{~mm}$. All anthropometric measures were measured to one decimal. Waist circumference was measured at the approximate midpoint between the lower margin of the last palpable rib and the top of the iliac crest. Hip circumference was measured around the widest portion of the buttocks. Body weight was measured using electronic calibrated scales to the nearest 100 grams, while subjects wore minimal clothing. Each measurement was repeated twice.

2.4. Statistical Analysis. Data analysis was performed using SAS software (version 8.02, Cary, NC, USA). Data in tables were reported with mean value and a standard deviation. Normality of distribution was tested using the Shapiro-Wilks test. Group comparisons were done using Mann-Whitney test and Student's $t$-test (CI of 95\%). Correlation was used to explore the nature of relationships among variables. Curve estimation models were used to assess the relationships among anthropometric variables, EAT and SAT thickness. ROC analysis was used to determine predictive capabilities of anthropometric variables EAT and SAT thickness for CAD. Accepted statistical significance was for $p<0.05$.

\section{Results}

Subjects were assigned to CAD group $(n=53)$ if they had $\geq 50 \%$ narrowing of one or more coronary arteries or to control, non-CAD group $(n=42)$, in which different valvular abnormalities were established. Subjects in the groups were of similar age and BMI. Larger values of waist circumference (WC), hip circumference (HC), waist-to-hip ratio (WHR), waist-to-height ratio (WHtR), EAT thickness, and SAT thickness were observed in CAD patients (Table 1).

Epicardial adipose tissue thickness showed weak to moderate correlation with $\mathrm{WC}$ and $\mathrm{HC}$ and weak correlation with BMI, WHtR, and SAT, while SAT showed stronger correlation with BMI, WC, HC, and WHtR. Neither EAT nor SAT correlated with age and WHR. 
TABLE 2: Receiver Operating Characteristic (ROC) analysis for prediction of coronary artery disease (CAD) with anthropometric measures and ultrasonographically obtained epicaradial and subcutaneous adipose tissue thickness.

\begin{tabular}{lccc}
\hline Tested variable & AUC & $p$ & $95 \%$ CI \\
\hline BMI & 0.605 & 0.082 & $0.481-0.729$ \\
Waist circumference & 0.688 & 0.002 & $0.579-0.797$ \\
Hip circumference & 0.643 & 0.018 & $0.528-0.758$ \\
Waist-to-hip ratio & 0.657 & 0.009 & $0.545-0.770$ \\
Waist-to-height ratio & 0.673 & 0.004 & $0.559-0.786$ \\
EAT thickness & 0.658 & 0.009 & $0.548-0.768$ \\
SAT thickness & 0.634 & 0.027 & $0.511-0.756$ \\
\hline Overall model & 0.751 & $<0.001$ & $0.651-0.834$ \\
\hline
\end{tabular}

Overall logistic regression model for CAD classification with anthropometric measures and EAT and SAT as predictor variables was tested with ROC analysis. AUC: area under curve; CI: confidence interval; BMI: body mass index; EAT: epicardial adipose tissue; SAT: subcutaneous adipose tissue. Coronary artery disease (CAD) was defined as $\geq 50 \%$ narrowing of one or more arteries.

3.1. Nonlinear Relationships between the Variables. Relationship between anthropometric variables (except WHR) and EAT thickness was described the best as a nonlinear $S$ curve relationship (Figures 1(a)-1(e)). An $S$ curve model was also the best fit for EAT and SAT thicknesses' relationship (Figure 1(f)). Visual inspection of scatter plots revealed that SAT thinner than $10 \mathrm{~mm}$ had a different relationship with EAT than SAT thicker than $10 \mathrm{~mm}$. Relationship between SAT thinner than $10 \mathrm{~mm}$ and EAT thickness was described the best as power curve relationship, since the power curve model explained $65.1 \%$ of variance $(p<0.001)$ (Figure 2$)$. When SAT thickness was above $10 \mathrm{~mm}$, no relationship with EAT thickness was established, as all regression models were nonsignificant (Figure 2).

3.2. Receiver Operating Characteristic Analysis. Receiver operating characteristic (ROC) curves for CAD classification with anthropometric variables and EAT, SAT thickness showed that all predictor variables, except BMI $(p=0.082)$, can detect CAD $(p<0.05)$, but with poor accuracy and similar sensitivity and specificity. Anthropometric variables and EAT and SAT had similar areas under curve. Using logistic regression for CAD prediction, overall model with anthropometric measures, SAT and EAT thickness as predictor variables were created and tested using ROC analysis. The overall model had moderate accuracy and the largest area under curve (AUC 0.751), while areas under curve for assessed measures ranged from 0.605 to 0.688 (Table 2).

\section{Discussion}

In our study cohort, all anthropometric variables, except WHR, showed moderate positive correlations with ultrasound measures of EAT and SAT. Such finding is obvious, since energy intake and expenditure disproportion lead to accumulation of body fat at all locations but also means that used anthropometric variables cannot discriminate between
EAT and SAT. Lack of correlation and relationship of EAT and SAT with WHR might indicate that WHR is not valuable anthropometric index for prediction of EAT and SAT. It was also reported previously that several other anthropometric measures are superior for assessment of body fat distribution than WHR [13-15].

All anthropometric variables (except WHR) and SAT thickness, independently of each other, showed a nonlinear $S$ curve relationship with EAT thickness in all analyzed subjects (Figure 1). As classic anthropometric measures measure both VAT and SAT, observed nonlinear relationship of EAT and classic anthropometric measures might be a "blurred picture" of an S curve clearly evident in relationship between EAT and SAT. Interestingly, in patients with SAT thinner than $10 \mathrm{~mm}$, we observed strong nonlinear power curve relationship with EAT (explaining $65.1 \%$ of the variance), while SAT thicker than $10 \mathrm{~mm}$ is independent of EAT thickness, since we could not establish any linear or nonlinear relationship (Figure 2). This unexpected findings, along with the average maximum thickness of EAT $(5.25 \pm 2.50 \mathrm{~mm})$, indicate that EAT does not accumulate above certain thickness regardless of increase in total adipose tissue volume.

Adipocytes in different compartments of adipose tissue enlarge by storing excess lipids, but when certain threshold overall volume of adipose tissue is reached, they cannot accumulate lipids anymore. It was shown that every person enters early adulthood with specific number of adipocytes, which can only be hypertrophy when accumulation of lipids occurs, while hyperplasia of precursors is strictly regulated [16]. Therefore, it is reasonable to predict that certain volume of adipose tissue has corresponding threshold values of different anthropometric measures.

Adipose tissue expands and undergoes extensive remodeling during positive caloric balance [16]. Adipose tissue remodeling occurs under inflammation and is characterized with relatively inadequate angiogenic remodeling and accumulation of extracellular matrix and immune cells [16]. It was reported that hypertrophy of the adipose tissue is associated with inability of recruitment and/or differentiation of existing preadipocytes into mature adipocytes, leading to overfill of adipocytes with lipids (lipid spillover) [17, 18]. Lipid spillover, through release of free fatty acids, acts as an inductor of endoplasmic stress and toll-like receptor type 4 pathways, responsible for the inflammation of the adipose tissue [19]. The enlargement of adipocytes leads to macrophage infiltration $[17,18,20]$. Curat et al. [21] showed, in vitro, that leptin (mostly produced by SAT) was responsible for macrophage extravasation, important step in initiation of adipose tissue inflammation [22]. Along with lipid spillover and leptin secretion, adipocyte hypertrophy is associated with hypoxia, a known inductor of inflammation [23]. Hypoxia acts through hypoxia induced factor 1 , transcription factor that leads to extensive extracellular remodeling of adipose tissue, thus hampering angiogenesis, which is a rate limiting step in adipose tissue expansion [24]. Hypoxia occurs as adipocyte grows above $150-200 \mu \mathrm{m}$ in diameter, thus exciding oxygen diffusion distance [25]. These pathophysiological processes could explain observed nonlinear relationship of EAT and other measures, indicating that EAT undergoes 


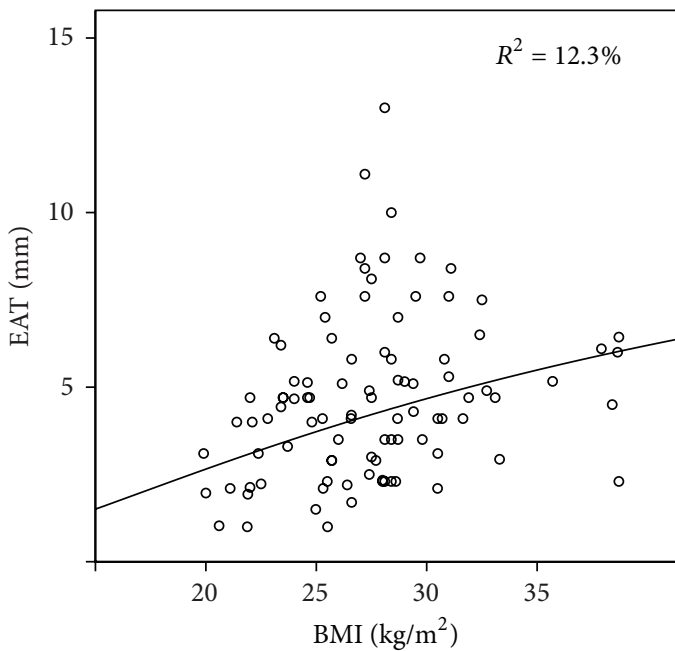

(a)

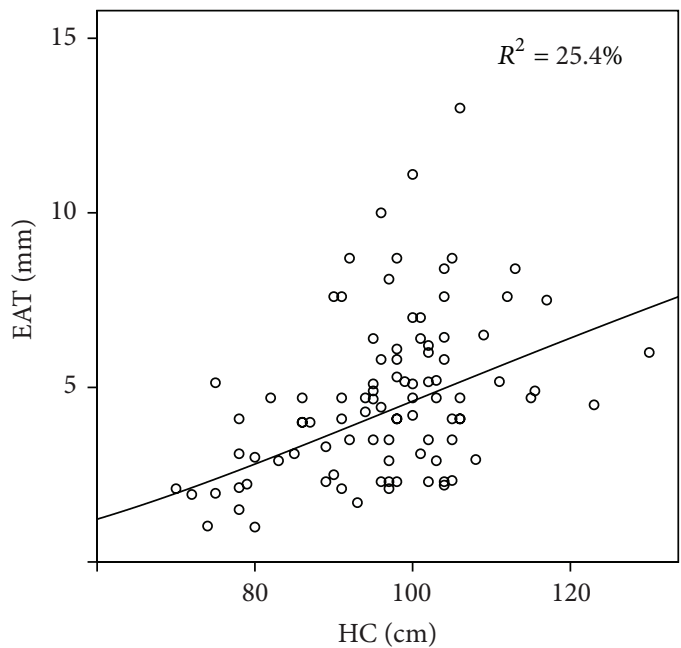

(c)

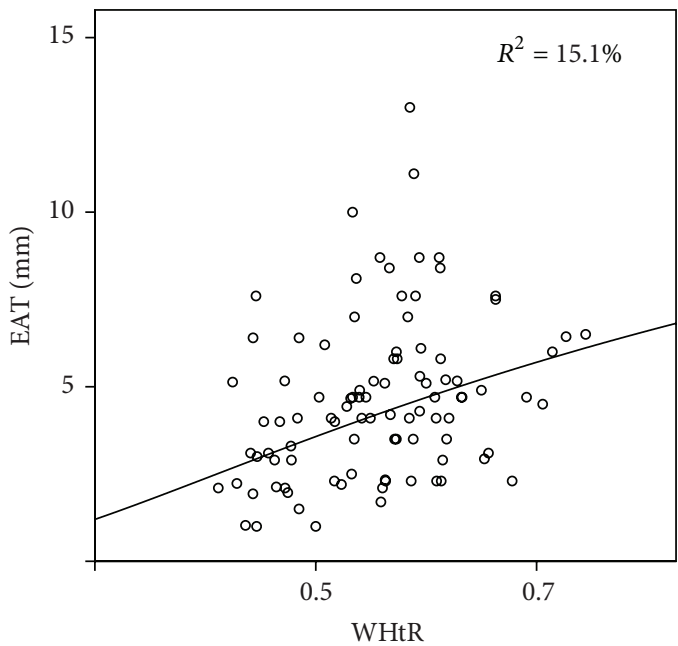

(e)

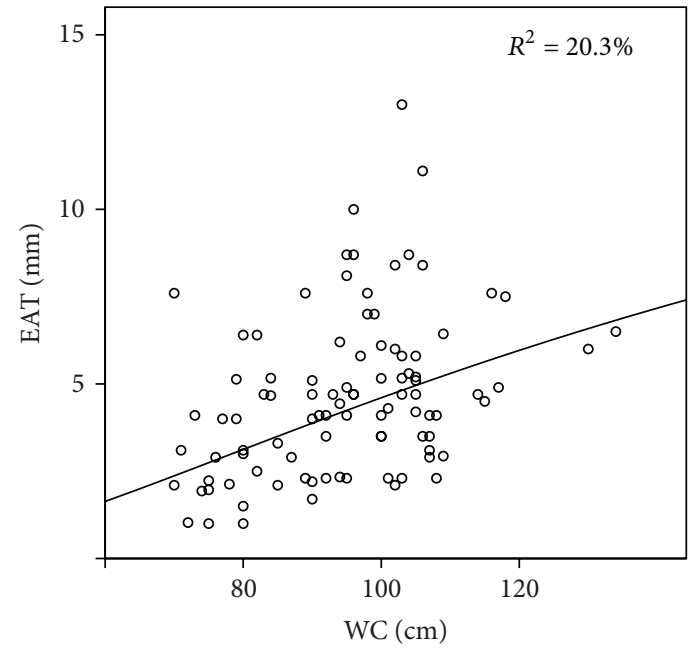

(b)

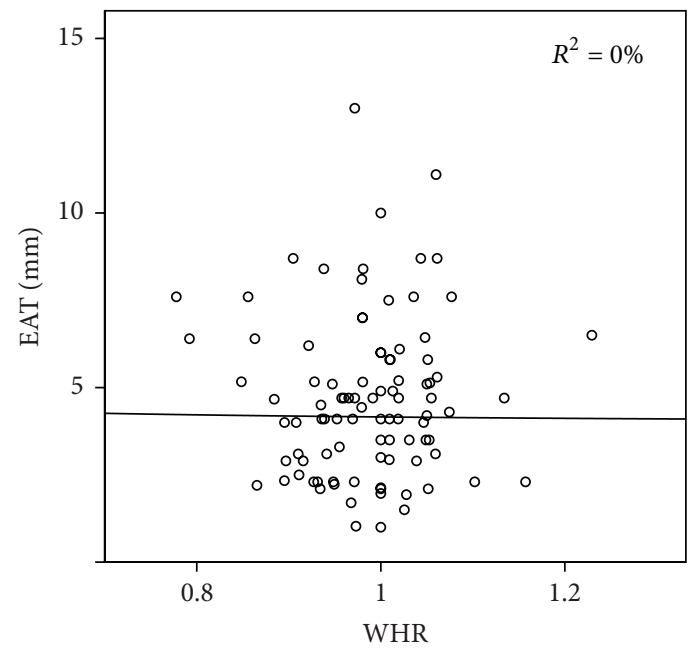

(d)

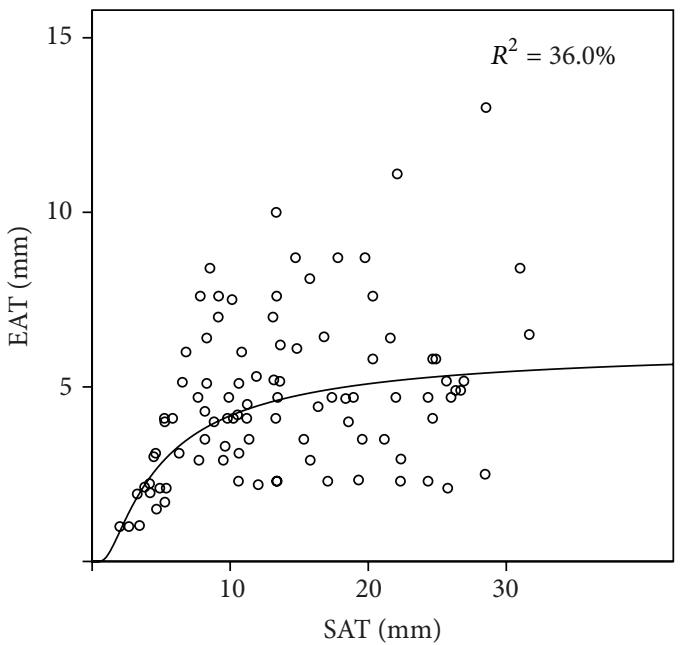

(f)

FIGURE 1: Nonlinear $S$ curve relationship of EAT thickness with anthropometric measures and SAT thickness. 


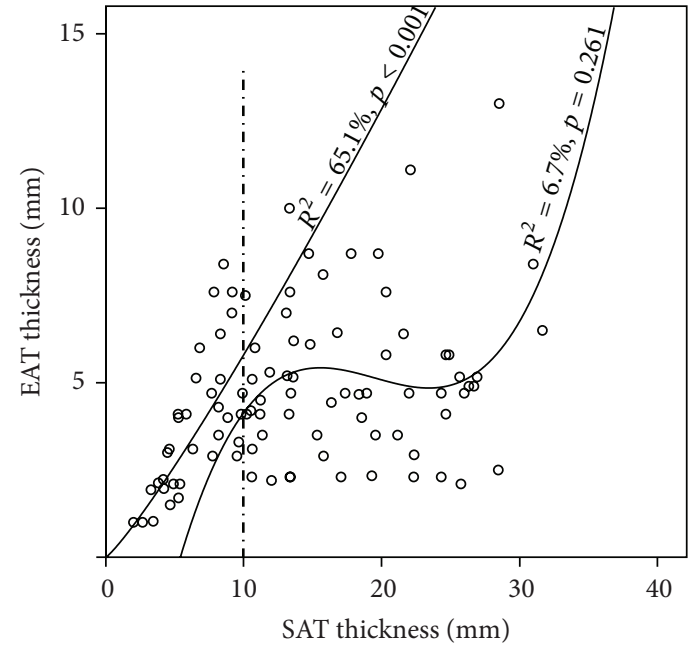

FIGURE 2: Nonlinear power curve relationship of EAT thickness and SAT thinner than $10 \mathrm{~mm}$.

remodeling and inflammation, thus hindering its ability to expand.

Moreover, accumulation of total adipose tissue leads to general low-grade inflammation, resulting in development of atherosclerosis [26]. Inflammation and atherosclerosis are the result of interplay of different cytokines, with important role of adipocytokines secreted by adipose tissue [26]. Coronarography is considered as standard for diagnosing CAD. Since it has several disadvantages and complications, complementary methods for assessing status of the coronary arteries would be useful. Anthropometric measures and SAT and EAT had poor accuracy at discriminating between CAD and controls in our study sample. The combined, overall model of anthropometric measures and SAT and EAT thickness had moderate accuracy at discriminating CAD and non-CAD patients. Since increased EAT thickness has been linked to CAD [3, 4, 11, 12], EAT thickness was expected to classify CAD patients better. Poor predictive accuracy of EAT thickness for CAD might be explained with current study setting, where control group consisted of patients with valvular diseases as controls. Recently, atherosclerotic pathophysiological process that results in CAD was associated with the aortic stenosis, the most common valvular disease [27]. Visceral obesity and its proinflammatory adipocytokines and subsequent inflammation have been linked with aortic stenosis, so called valvulometabolic risk [28]. Decreased levels of anti-inflammatory adiponectin have been linked with greater valvular inflammatory activity [29], while increased levels of leptin have been found in blood of the valvular patients [30]. Systemic effects of different compartments of adipose tissue could contribute to development of aortic stenosis and other valvular disease, as well as potential paracrine effects of the EAT and periaortic adipose tissue. Therefore, similar pathophysiological background of CAD and valvular disease might explain poor predictive accuracy of EAT thickness in our study sample, where the proportion of our control patients had aortic stenosis. Nevertheless, further research on role of EAT is warranted, since, in healthy individuals, it protects the myocardium while its expansion might lead to lipotoxicity and generation of proinflammatory cytokines [31]. Imaging MRI and echocardiographic studies showed that myocardial fat and cardiac dysfunction (i.e., left ventricle LV overload, systolic dysfunction, and hypertrophy) are associated with accumulation of EAT [32, 33]. Our study is limited to lack of expression profile of inflammation markers in adipose tissue and blood, as well as a relatively small sample size.

In conclusion, in cardiac patients, the EAT thickness follows a nonlinear relationship with anthropometric measures and SAT thickness. Ultrasound measures of EAT thickness have similar predictive accuracy for CAD to anthropometric measures of visceral obesity.

\section{Conflict of Interests}

The authors declare that there is no conflict of interests.

\section{References}

[1] W. Shen, Z. Wang, M. Punyanita et al., "Adipose tissue quantification by imaging methods: a proposed classification," Obesity Research, vol. 11, no. 1, pp. 5-16, 2003.

[2] K. Maeda, K. Okubo, I. Shimomura, K. Mizuno, Y. Matsuzawa, and K. Matsubara, "Analysis of an expression profile of genes in the human adipose tissue," Gene, vol. 190, no. 2, pp. 227-235, 1997.

[3] G. Iacobellis, F. Assael, M. C. Ribaudo et al., "Epicardial fat from echocardiography: a new method for visceral adipose tissue prediction," Obesity Research, vol. 11, no. 2, pp. 304-310, 2003.

[4] G. Iacobellis, D. Corradi, and A. M. Sharma, "Epicardial adipose tissue: anatomic, biomolecular and clinical relationships with the heart," Nature Clinical Practice Cardiovascular Medicine, vol. 2, no. 10, pp. 536-543, 2005.

[5] J.-P. Després, I. Lemieux, J. Bergeron et al., "Abdominal obesity and the metabolic syndrome: contribution to global cardiometabolic risk," Arteriosclerosis, Thrombosis, and Vascular Biology, vol. 28, no. 6, pp. 1039-1049, 2008.

[6] C. S. Fox, J. M. Massaro, U. Hoffmann et al., "Abdominal visceral and subcutaneous adipose tissue compartments: association with metabolic risk factors in the framingham heart study," Circulation, vol. 116, no. 1, pp. 39-48, 2007.

[7] G. L. Vega, B. Adams-Huet, R. Peshock, D. Willett, B. Shah, and S. M. Grundy, "Influence of body fat content and distribution on variation in metabolic risk," Journal of Clinical Endocrinology of Metabolism, vol. 91, no. 11, pp. 4459-4466, 2006.

[8] K. Azuma, L. K. Heilbronn, J. B. Albu, S. R. Smith, E. Ravussin, and D. E. Kelley, "Adipose tissue distribution in relation to insulin resistance in type 2 diabetes mellitus," The American Journal of Physiology_Endocrinology and Metabolism, vol. 293, no. 1, pp. E435-E442, 2007.

[9] K. G. M. M. Alberti, P. Zimmet, and J. Shaw, "Metabolic syndrome-a new world-wide definition. A consensus statement from the International Diabetes Federation," Diabetic Medicine, vol. 23, no. 5, pp. 469-480, 2006.

[10] S. J. Kim, H. S. Kim, J. W. Jung, N. S. Kim, C. I. Noh, and Y. M. Hong, "Correlation between epicardial fat thickness by echocardiography and other parameters in obese adolescents," Korean Circulation Journal, vol. 42, no. 7, pp. 471-478, 2012. 
[11] S. Eroglu, L. E. Sade, A. Yildirir et al., "Epicardial adipose tissue thickness by echocardiography is a marker for the presence and severity of coronary artery disease," Nutrition, Metabolism and Cardiovascular Diseases, vol. 19, no. 3, pp. 211-217, 2009.

[12] G. Iacobellis, H. J. Willens, G. Barbaro, and A. M. Sharma, "Threshold values of high-risk echocardiographic epicardial fat thickness," Obesity, vol. 16, no. 4, pp. 887-892, 2008.

[13] B. L. Wajchenberg, "Subcutaneous and visceral adipose tissue: their relation to the metabolic syndrome," Endocrine Reviews, vol. 21, no. 6, pp. 697-738, 2000.

[14] M. Ashwell, P. Gunn, and S. Gibson, "Waist-to-height ratio is a better screening tool than waist circumference and BMI for adult cardiometabolic risk factors: systematic review and metaanalysis," Obesity Reviews, vol. 13, no. 3, pp. 275-286, 2012.

[15] G. H. Goossens, "The role of adipose tissue dysfunction in the pathogenesis of obesity-related insulin resistance," Physiology and Behavior, vol. 94, no. 2, pp. 206-218, 2008.

[16] K. Sun, C. M. Kusminski, and P. E. Scherer, "Adipose tissue remodeling and obesity," The Journal of Clinical Investigation, vol. 121, no. 6, pp. 2094-2101, 2011.

[17] A. Hammarstedt, T. E. Graham, and B. B. Kahn, "Adipose tissue dysregulation and reduced insulin sensitivity in non-obese individuals with enlarged abdominal adipose cells," Diabetology \& Metabolic Syndrome, vol. 4, no. 1, article 42, 2012.

[18] P. Isakson, A. Hammarstedt, B. Gustafson, and U. Smith, "Impaired preadipocyte differentiation in human abdominal obesity: role of Wnt, tumor necrosis factor- $\alpha$, and inflammation," Diabetes, vol. 58, no. 7, pp. 1550-1557, 2009.

[19] H. S. Schipper, B. Prakken, E. Kalkhoven, and M. Boes, "Adipose tissue-resident immune cells: key players in immunometabolism," Trends in Endocrinology \& Metabolism, vol. 23, no. 8, pp. 407-415, 2012.

[20] B. Gustafson, S. Gogg, S. Hedjazifar, L. Jenndahl, A. Hammarstedt, and U. Smith, "Inflammation and impaired adipogenesis in hypertrophic obesity in man," American Journal of PhysiologyEndocrinology and Metabolism, vol. 297, no. 5, pp. E999-E1003, 2009.

[21] C. A. Curat, A. Miranville, C. Sengenès et al., "From blood monocytes to adipose tissue-resident macrophages: induction of diapedesis by human mature adipocytes," Diabetes, vol. 53, no. 5, pp. 1285-1292, 2004.

[22] A. Taube, R. Schlich, H. Sell, K. Eckardt, and J. Eckel, "Inflammation and metabolic dysfunction: links to cardiovascular diseases," The American Journal of Physiology-Heart and Circulatory Physiology, vol. 302, no. 11, pp. H2148-H2165, 2012.

[23] P. Trayhurn and I. S. Wood, "Adipokines: inflammation and the pleiotropic role of white adipose tissue," British Journal of Nutrition, vol. 92, no. 3, pp. 347-355, 2004.

[24] M. A. Rupnick, D. Panigrahy, C.-Y. Zhang et al., "Adipose tissue mass can be regulated through the vasculature," Proceedings of the National Academy of Sciences of the United States of America, vol. 99, no. 16, pp. 10730-10735, 2002.

[25] M. C. Brahimi-Horn, J. Chiche, and J. Pouysségur, "Hypoxia signalling controls metabolic demand," Current Opinion in Cell Biology, vol. 19, no. 2, pp. 223-229, 2007.

[26] U. Salmenniemi, E. Ruotsalainen, J. Pihlajamäki et al., "Multiple abnormalities in glucose and energy metabolism and coordinated changes in levels of adiponectin, cytokines, and adhesion molecules in subjects with metabolic syndrome," Circulation, vol. 110, no. 25, pp. 3842-3848, 2004.
[27] N. M. Rajamannan, R. O. Bonow, and S. H. Rahimtoola, "Calcific aortic stenosis: an update," Nature Clinical Practice Cardiovascular Medicine, vol. 4, no. 5, pp. 254-262, 2007.

[28] P. Mathieu, J. P. Després, and P. Pibarot, "The 'valvulometabolic' risk in calcific aortic valve disease," Canadian Journal of Cardiology, vol. 23, pp. 32B-39B, 2007.

[29] D. Mohty, P. Pibarot, N. Côté et al., "Hypoadiponectinemia is associated with valvular inflammation and faster disease progression in patients with aortic stenosis," Cardiology, vol. 118, no. 2, pp. 140-146, 2011.

[30] E. Beckmann, J. B. Grau, R. Sainger, P. Poggio, and G. Ferrari, "Insights into the use of biomarkers in calcific aortic valve disease," The Journal of Heart Valve Disease, vol. 19, no. 4, pp. 441-452, 2010.

[31] P. Iozzo, "Myocardial, perivascular,and epicardial fat," Diabetes Care, vol. 34, supplement 2, pp. S371-S379, 2011.

[32] M. Kankaanpää, H.-R. Lehto, J. P. Pärkkä et al., "Myocardial triglyceride content and epicardial fat mass in human obesity: relationship to left ventricular function and serum free fatty acid levels," Journal of Clinical Endocrinology and Metabolism, vol. 91, no. 11, pp. 4689-4695, 2006.

[33] G. Pucci, F. Battista, S. de Vuono et al., "Pericardial fat, insulin resistance, and left ventricular structure and function in morbid obesity," Nutrition, Metabolism and Cardiovascular Diseases, vol. 24, no. 4, pp. 440-446, 2014. 


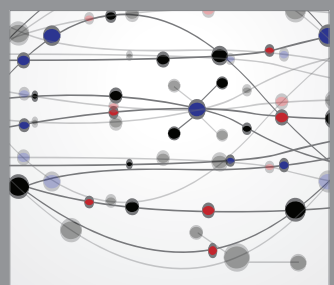

The Scientific World Journal
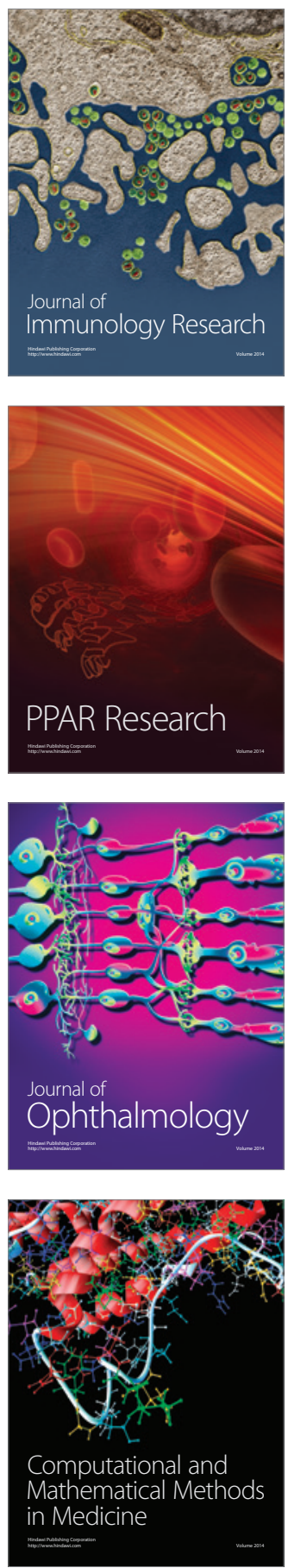

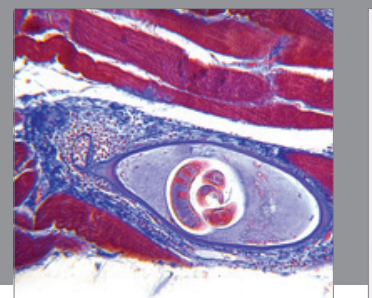

Gastroenterology

Research and Practice
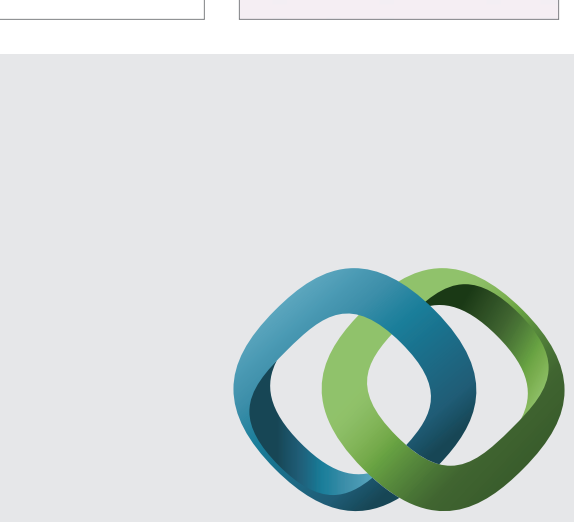

\section{Hindawi}

Submit your manuscripts at

http://www.hindawi.com
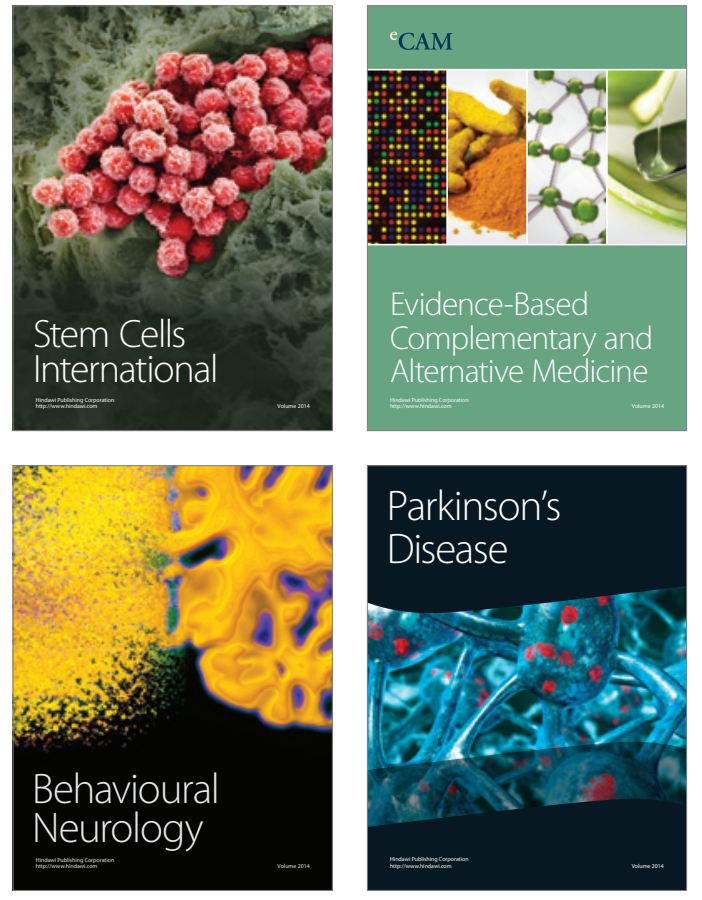
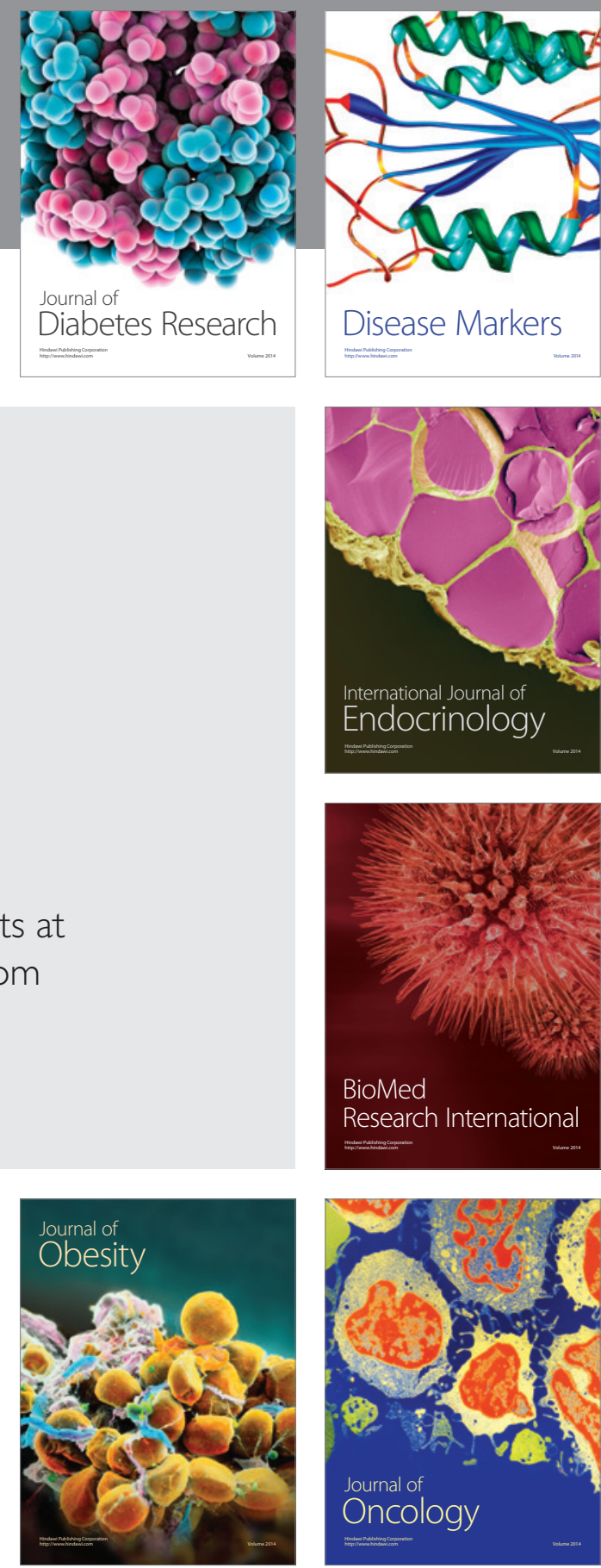

Disease Markers
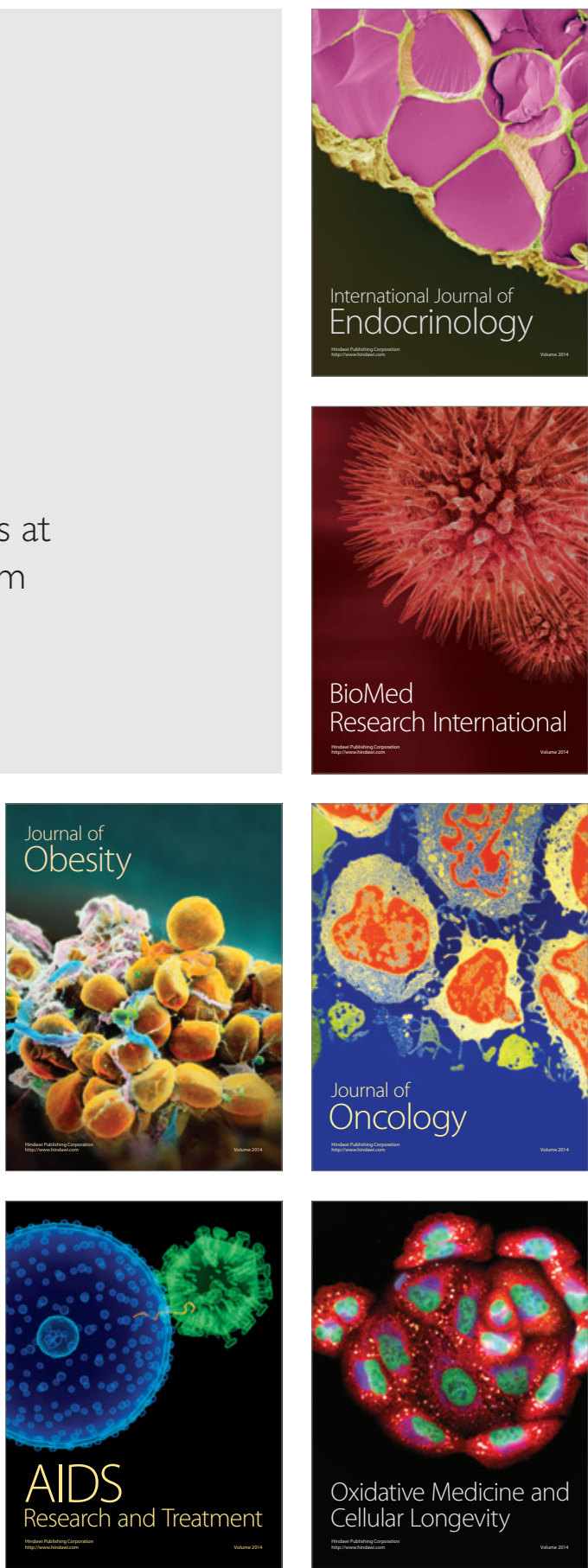